\title{
Surgical and Conservative Management of Malignant Bowel Obstruction: Outcome and Prognostic Factors
}

This article was published in the following Dove Press journal: Cancer Management and Research

\author{
Kaiying $\mathrm{Yu}^{1-3, *}$ \\ Lihui Liu ${ }^{3,4, *}$ \\ Xiaowei Zhang ${ }^{1-3}$ \\ Zhanzhi Zhang ${ }^{1-3}$ \\ Benqiang Rao ${ }^{1-3}$ \\ Yongbing Chen ${ }^{1-3}$ \\ Suyun $\mathrm{Li}^{2,3}$ \\ Hanping Shi ${ }^{1-3}$
}

'Department of General Surgery, Beijing Shijitan Hospital, Capital Medical University, Beijing 100038, People's Republic of China; ${ }^{2}$ Department of Clinical Nutrition, Beijing Shijitan Hospital, Capital Medical University, Beijing 100038, People's Republic of China; ${ }^{3}$ Ninth School of Clinical Medicine, Peking University, Beijing 100038, People's Republic of China; ${ }^{4}$ Department of Nursing, Beijing Shijitan Hospital, Capital Medical University, Beijing 100038, People's Republic of China

*These authors contributed equally to this work

Correspondence: Hanping Shi Department of General Surgery, Beijing Shijitan Hospital, Capital Medical University, No. I0th Tieyi Road, Haidian District, Beijing I00038, People's Republic of China

Tel +86- | 380274| 263

Fax +86-010-63926519

Email shihp@vip.l63.com
Purpose: Malignant bowel obstruction (MBO) is a common problem in late-stage cancer patients. Many factors are suggested to be associated with the prognosis of MBO. The current investigation was designed to explore the factors associated with the prognosis of conservative and surgery treatment in one single institution.

Patients and Methods: Sixty-four patients of MBO were recruited into the study. Demographic and clinical data including gender, age, primary cancer, radiological and laboratory examinations, and nutritional and pain index scaling were extracted for further analysis. Kaplan-Meier analysis and logistic regression analysis were used to compare the prognosis and detect significant factors.

Results: Of the 64 patients, there is no statistical difference in baseline features between conservative and surgical group. However, the length of stay, total medical costs, readmission interval, and re-admission rate are statistically significant. There is no significance in Kaplan-Meier log rank test for median survival time, though the overall survival time in the conservative group is longer than that of the surgery group. Logistic regression analysis has found that prior chemotherapy is a significant predictor for final survival outcome.

Conclusion: The election of surgery might not improve the overall survival time. Nonsurgical procedures, especially chemotherapy, might be preferable for MBO patients.

Keywords: malignant bowel obstruction, surgical treatment, conservative management, prognosis, survival

\section{Introduction}

Malignant bowel obstruction (MBO) is a common clinical problem, especially in terminal malignant cancer patients. ${ }^{1}$ Although the exact definition of MBO varies, one generally accepted version constitutes clinical symptoms of bowel obstruction concomitant with evidence of primary or secondary intra-abdominal cancer. ${ }^{2} \mathrm{MBO}$ may occur as a result of cancer advancement (eg, tumor over-growth), anti-cancer treatment (eg, radiation scarring), or other benign factors (eg, adhesive blockade). In later stages, the obstructed intestinal segments may impede the passage of intraluminal contents, resulting in imbalance of electrolyte or even mesenteric ischemia. $^{3}$

The overall incidence of $\mathrm{MBO}$ in cancer patients has been reported to range from $3 \%$ to $15 \%$, with colorectal $(25-40 \%)^{4}$ and ovarian cancer $(16-22 \%)^{5}$ being the most common. Coincidentally, the top two primary cancers in MBO patients are also of colorectal and gynecological origins. ${ }^{6}$ The diagnosis of MBO mainly relies 
on radiological examinations apart from an established disease history. Standing plain X-ray with contrast agent may exhibit the site and degree of obstruction. ${ }^{7}$ Distention of the proximal intestine loop with air-fluid levels is a typical sign in imaging tests. In addition, computed tomography can achieve a remarkably higher specificity of approximately $100 \%$ in determining the obstruction level. ${ }^{8}$ The acquired information is necessary for the decision-making process regarding choosing either radical or conservative treatments.

The main treatment of MBO includes palliative surgery, self-expanding stents, nasogastric tube, and palliative pharmacological options. ${ }^{9,10}$ The goals of these treatments are to re-establish the digestive flow, relieve compressing symptoms, and recover nutritional status. No matter the strategy, the outcome and long-term prognosis are not always as satisfactory as expected in many cases. For instance, a reported median post-operative survival time was merely 191 days, ${ }^{11}$ and the mean survival for advanced inoperable MBO patients was less than 4-5 weeks. ${ }^{4}$ This might be due to the worsened general status and advancement of cancer progression. Many factors, such as age, nutrition, tumor staging, and perioperative performance, are associated with the prognosis. ${ }^{12}$

In this article, we retrospectively reviewed MBO cases in our department. The authors herein described factors associated with $\mathrm{MBO}$, including demographics, primary cancer site, management, and tumor features. We also conducted the follow-up and evaluated the prognosis of conservative or operative patients.

\section{Patients and Methods}

\section{Patients Selection}

Patients who were admitted between June 2017 and October 2019 into the department of general surgery of Beijing Shijitan Hospital (Beijing, China) were retrospectively reviewed for $\mathrm{MBO}$ according to the definition proposed by a clinical protocol committee: 1) clinical evidence of bowel obstruction via history, physical, or radiological examinations; 2) obstruction beyond the Treitz ligament; and 3) intra-abdominal primary cancer with incurable disease or non-intra-abdominal primary cancer with clear intra-peritoneal disease. ${ }^{13}$ Nonmalignant related obstructions, such as adhesion due to previous non-cancer surgery, intussusception, hernia, inflammation, fecalith, volvulus, enterospasm, and mesenteric ischemia, were not included in the current study. This study was approved by the Beijing Shijitan Hospital ethics committee and was in accordance with the principles of the Declaration of Helsinki. Written informed consent was obtained from the patients or the authorized person. The included patients were further reviewed to extract their clinical data, such as demographic information, physical examinations, radiological images, laboratory results, surgical findings, histological reports, nutritional status, etc. Patient-generated subjective global assessment (PG-SGA) score, Karnofsky performance score (KPS), visual analog scale (VAS), modified frailty index ( $\mathrm{mFI})$, the American Society of Anesthesiology (ASA) score, and Charlson comorbidity index (CCI) had been evaluated according to the previously published methods. ${ }^{14-18}$ Indication for surgery mainly included failure of conservative therapy. The procedure of the surgery consisted of tumor resection or stoma, depending on specific intra-operative intraabdominal conditions.

\section{Statistical Analysis}

All statistical differences were calculated using SPSS 20.0 for windows. Continuous data were performed for the Shapiro-Wilk test to detect the distribution. For normally distributed data, student $t$ test was adopted; otherwise Mann-Whitney U-test was used. Chi-square test was used to compare the difference of numeration data. Survival analysis was conducted with Kaplan-Meier method (Log rank tests). Logistic regression analysis was performed with "forward LR" method. A $P$-value less than 0.05 was considered statistically significant.

\section{Results \\ Demographic Data}

We identified a total of 64 patients (27 male and 37 female) meeting our inclusion criteria. Demographic information is summarized in Table 1. Of the 64 patients, $16(25 \%)$ received conservative treatment, while $48(75 \%)$ received surgical treatment. There is no statistical significance in the comparison of baseline data, including gender distribution, age, BMI, and marital status, between conservative and surgical group ( $P>0.05$ for all). All the 64 patients are at the late stages of cancer, consistent with previous reports. The PGSGA, KPS, VAS, ASA, mFI, and CCI scores are insignificantly different between the two groups ( $P>0.05$ for all). In the compared variables, only the total length of stay, total medical costs, re-admission interval, and re-admission rate are of statistical significance $(P<0.05)$. The hospital stay time 
Table I Demographic and Clinical Characteristics of Patients with MBO. A Total of 64 MBO Patients Were Recruited into the Analysis. The Patients Were Divided into Conservative or Surgery Group. Data Were Expressed as Mean \pm SD or Percentage

\begin{tabular}{|c|c|c|c|c|}
\hline & Overall $(n=64)$ & $\begin{array}{l}\text { Palliative Treatment } \\
(n=16)\end{array}$ & $\begin{array}{l}\text { Surgical Treatment } \\
(n=48)\end{array}$ & $P$-value \\
\hline \multicolumn{5}{|l|}{ Gender } \\
\hline Male, n (\%) & 27 (42\%) & 7 & 20 & 1.00 \\
\hline Female, n (\%) & $37(58 \%)$ & 9 & 28 & \\
\hline Age at admission, year, median (range) & $55.5(2 \mid-87)$ & $56(21-86)$ & $55.5(27-87)$ & 0.93 \\
\hline $\mathrm{BMI}, \mathrm{kg} / \mathrm{m}^{2}$ & 22.4 & 21.6 & 22.7 & 0.26 \\
\hline \multicolumn{5}{|l|}{ Marital status } \\
\hline Married, n (\%) & $62(97 \%)$ & 15 (94\%) & 47 (98\%) & 0.41 \\
\hline Unmarried, n (\%) & $2(3 \%)$ & $\mathrm{I}(6 \%)$ & I (2\%) & \\
\hline Total length of stay, days (median) & 23.5 & 10.0 & 29.5 & $9.23 \mathrm{E}-09$ \\
\hline Post-operative length of stay, days (median) & $(-)$ & $(-)$ & 17 & $(-)$ \\
\hline \multicolumn{5}{|l|}{ Primary tumor location } \\
\hline Stomach & 7 & 3 & 4 & 0.60 \\
\hline Small intestine & 3 & 0 & 3 & \\
\hline Pancreas & 2 & 0 & 2 & \\
\hline Gallbladder & 1 & 1 & 0 & \\
\hline Colon & 23 & 4 & 19 & \\
\hline Ovary & 4 & I & 3 & \\
\hline Uterus & 5 & 2 & 3 & \\
\hline Kidney & 2 & I & I & \\
\hline Bladder & 2 & 1 & I & \\
\hline Peritoneum & 9 & 2 & 7 & \\
\hline Retroperitoneum & 1 & 0 & I & \\
\hline Unclear & 5 & 1 & 4 & \\
\hline Ascites, n (\%) & $29(45 \%)$ & 7 (44\%) & $22(46 \%)$ & 1.00 \\
\hline Prior chemotherapy, n (\%) & $42(66 \%)$ & 12 (75\%) & $30(63 \%)$ & 0.54 \\
\hline Prior radiotherapy, n (\%) & II (I7\%) & $3(19 \%)$ & $8(17 \%)$ & 1.00 \\
\hline Surgery, n (\%) & 48 (75\%) & $(-)$ & 48 (100\%) & $(-)$ \\
\hline Tumor resection & 34 (53\%) & $(-)$ & 34 (7।\%) & $(-)$ \\
\hline Stoma & $16(25 \%)$ & $(-)$ & $16(33 \%)$ & $(-)$ \\
\hline Post-operative anastomotic leakage, $\mathrm{n}(\%)$ & $(-)$ & $(-)$ & $7(15 \%)$ & $(-)$ \\
\hline Surgical site infection, $\mathrm{n}(\%)$ & $(-)$ & $(-)$ & $3(6 \%)$ & $(-)$ \\
\hline \multicolumn{5}{|l|}{ cTNM staging, n (\%) } \\
\hline I & 0 & 0 & 0 & 1.00 \\
\hline II & 0 & 0 & 0 & \\
\hline III & $5(8 \%)$ & I (6\%) & $4(8 \%)$ & \\
\hline IV & 59 (92\%) & 15 (94\%) & 44 (92\%) & \\
\hline Blood loss during surgery, $\mathrm{mL}$ (mean $\pm \mathrm{SD}$ ) & $(-)$ & $(-)$ & $227 \pm 284$ & $(-)$ \\
\hline Surgery duration, $\mathrm{h}($ mean $\pm \mathrm{SD})$ & $(-)$ & $(-)$ & $4.5 \pm 3.1$ & $(-)$ \\
\hline PG-SGA scoring at admission (mean \pm SD) & $11.7 \pm 6.2$ & $12.8 \pm 7.6$ & $11.3 \pm 5.9$ & 0.37 \\
\hline PG-SGA scoring at discharge (mean $\pm S D)$ & $9.1 \pm 5.0$ & $9.7 \pm 6.0$ & $8.9 \pm 4.8$ & 0.55 \\
\hline KPS score at admission (mean \pm SD) & $69.7 \pm 32.3$ & $65.0 \pm 34.9$ & $71.1 \pm 3.3$ & 0.44 \\
\hline KPS score at discharge $($ mean \pm SD) & $66.9 \pm 35.7$ & $69.6 \pm 38.7$ & $66.2 \pm 35.7$ & 0.73 \\
\hline Total medical costs, $¥($ mean $\pm S D)$ & $103,335.9 \pm 80,01 \mid .2$ & $|8,567.| \pm|2,0| 0.3$ & $131,592.1 \pm 73,723.0$ & I.44E- 14 \\
\hline TPN, n (\%) & $4 \mid(64 \%)$ & $8(50 \%)$ & $33(69 \%)$ & 0.23 \\
\hline EN, n (\%) & 57 (89\%) & $13(8 \mid \%)$ & 44 (92\%) & 0.49 \\
\hline
\end{tabular}


Table I (Continued).

\begin{tabular}{|c|c|c|c|c|}
\hline & Overall $(n=64)$ & $\begin{array}{l}\text { Palliative Treatment } \\
(n=16)\end{array}$ & $\begin{array}{l}\text { Surgical Treatment } \\
(n=48)\end{array}$ & $P$-value \\
\hline Hemoglobin, g/L (mean $\pm S D)$ & $108.3 \pm 25.8$ & $113.7 \pm 35.8$ & $106.3 \pm 25.5$ & 0.28 \\
\hline White blood cell, $10^{9} / \mathrm{L}($ mean $\pm S D)$ & $8.4 \pm 11.1$ & $\mid 1.8 \pm 21.4$ & $8.1 \pm 4.8$ & 0.79 \\
\hline Albumin, $g / L$ (mean $\pm S D)$ & $34.1 \pm 6.3$ & $35.1 \pm 9.5$ & $33.8 \pm 8.4$ & 0.88 \\
\hline VAS score $($ mean $\pm S D)$ & $4.5 \pm 1.9$ & $4.3 \pm 1.9$ & $4.6 \pm 2.0$ & 0.93 \\
\hline Modified Frailty Index (mean $\pm S D)$ & $2.7 \pm 2.0$ & $3.1 \pm 2.1$ & $2.6 \pm 1.9$ & 0.31 \\
\hline ASA score (mean $\pm S D)$ & $2.0 \pm 0.8$ & $1.9 \pm 1.0$ & $2.0 \pm 0.7$ & 0.56 \\
\hline Charlson Comorbidity Index (mean \pm SD) & $6.5 \pm 3.1$ & $6.1 \pm 3.0$ & $6.7 \pm 3.3$ & 0.55 \\
\hline Being able to take solid food after surgery, n (\%) & $(-)$ & $(-)$ & $31(65 \%)$ & $(-)$ \\
\hline $\begin{array}{l}\text { Post-operative time to take solid food, days (mean } \\
\pm S D \text { ) }\end{array}$ & $(-)$ & $(-)$ & $6.9 \pm 5.6$ & $(-)$ \\
\hline Post-operative 30-day mortality, n (\%) & $(-)$ & $(-)$ & $4(8 \%)$ & $(-)$ \\
\hline Re-admission interval, days (mean $\pm \mathrm{SD}$ ) & $14.7 \pm 6.6$ & $19.2 \pm 8.0$ & $12.0 \pm 6.3$ & 0.01 \\
\hline Re-admission rate, n (\%) & $25(39 \%)$ & $10(63 \%)$ & $15(31 \%)$ & 0.04 \\
\hline Post-operative survival time, days (mean $\pm \mathrm{SD}$ ) & $(-)$ & $(-)$ & $189.3 \pm 193.1$ & $(-)$ \\
\hline
\end{tabular}

in the treatment group is nearly three times that of the conservative group. The significance in the comparison of readmission rate is marginal $(P=0.04)$. For the surgery group, the post-operative 30 -day mortality rate is $8 \%$ (4/48). Since it has been reported that most of the MBO patients were of colon origins, ${ }^{4}$ we list the colon data separately in Supplementary Table S1.

\section{Survival Analysis}

The mean post-operative survival time in the surgery group is 189.3 days. The median overall survival time in the surgery and palliative group is 89 and 396 days, respectively (Table 2). For the entire cohort, the median survival time is 113 days. There is no significance in median survival time between two groups by KaplanMeier log rank test $(P=0.169)$ (Figure 1$)$.

\section{Logistic Regression Analysis}

In order to detect the variables associated with the survival status, binary logistic regression analysis was performed with dead or survived status as the ending point. In this study, since the overall sample size is 64 , up to four or five variables are statistically optimal to be recruited into the initial analysis. Therefore, five clinically remarkable variables, including prior chemotherapy, prior radiotherapy, ASA score, PG-SGA scoring at admission, and PG-SGA scoring at discharge were analyzed with logistic regression forward LR method. Consequently, two variables, including prior chemotherapy and PG-SGA scoring at discharge, were automatically remained in the equation (Table 3 ), and three variables (prior radiotherapy, ASA score, and PGSGA scoring at admission) were automatically dropped from the equation (Table 4). In brief, logistic regression analysis found that prior chemotherapy was significantly associated with the final surviving status $(P=0.032$, $\mathrm{OR}=12.443,95 \% \mathrm{CI}=1.247-124.132$ ).

\section{Discussion}

In the current investigation, demographic data of $\mathrm{MBO}$ patients, and the conservative and surgical management

Table 2 Overall Survival Time of Palliative and Surgery Group. Data Were Presented as Quartile (25\%, 50\%, and 75\%) and Standard Error (SE). Survival Time (Days) Was Calculated in Palliative, Surgery, or Overall Group, Respectively

\begin{tabular}{|l|l|l|l|l|l|l|}
\hline \multirow{2}{*}{ Group } & \multicolumn{2}{|l|}{$\mathbf{2 5 \%}$} & $\mathbf{5 0 \%}$ & $\mathbf{3}$ & \multicolumn{2}{l|}{} \\
\cline { 2 - 7 } & Estimate & SE & Estimate & SE & Estimate & SE \\
\hline Palliative group & 567 & 79.16 & 396 & 357.44 & 96 & 69.92 \\
Surgery group & 250 & 150.04 & 89 & 24.63 & 45 & 22.29 \\
All cohort & 396 & 134.81 & 113 & 28.70 & 62 & 22.87 \\
\hline
\end{tabular}




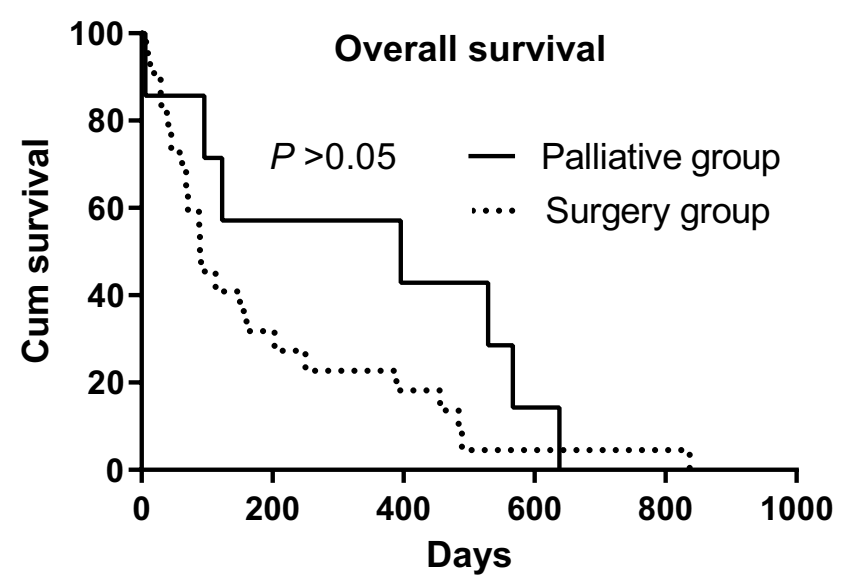

Figure I Overall survival for palliative and surgery groups of malignant bowel obstruction. $P$-values were estimated by Kaplan-Meier log rank test.

were compared and analyzed. Concisely, this study found that hospital length of stay and total medical costs were significantly higher, while re-admission interval and readmission rate were significantly lower in the surgical group than in the palliative group; Survival analysis indicated an insignificance of survival between palliative and surgery groups; Logistic regression analysis found that prior chemotherapy was a statistically significant predictor for survival status.

MBO is a tough problem for end-stage cancer patients in clinical practice. Generally, the possible treatment includes surgery and palliative therapy. The decisionmaking process calls for individualized assessment on the patient's overall body health status, expected survival time, and prior radiologic or chemotherapy history. In patients with good performance and well general status that can afford the devastation of surgical procedure, surgery has always been recommended to reestablish the gastrointestinal flow and relieve the digestive occlusion. This implies that surgical patients seem to be less "sick" than non-surgical patients. However, our finding is paradoxical with the reasoning in that the PG-SGA, KPS, VAS, ASA, mFI, and CCI scores have no statistical difference
Table 4 Variables Not in the Final Equation. Variables Were Analyzed Through the Use of Logistic Regression Analysis with Forward LR Method. Prior Radiotherapy Was Transformed to Binary Data $(0=$ no, I=yes)

\begin{tabular}{|l|l|l|l|}
\hline & Score & df & Sig. \\
\hline Prior radiotherapy & 0.379 & $\mathrm{I}$ & 0.538 \\
ASA score & 1.154 & $\mathrm{I}$ & 0.283 \\
PG-SGA scoring at admission & 0.944 & $\mathrm{I}$ & 0.331 \\
Overall statistics & 2.779 & 3 & 0.427 \\
\hline
\end{tabular}

between surgical and non-surgical patients. Furthermore, the survival time of surgical patients does not meet the expectation to be longer than non-surgical patients, suggesting that the non-surgical group is not "sicker" than the surgical group. The divergence is likely due to a sufficient nutritional support. The observation that patients undergoing surgery experienced a lower survival rate, though not statistical significant, indicated that surgery might not improve survival among MBO patients even though the surgical patients' general status was no worse than the non-surgical patients. Under this circumstance, there should be other factors potentially affecting the longterm prognosis. We found that a factor associated with final survival outcome is the prior chemotherapy by logistic regression analysis. This result is consistent with Chouhan's multivariate analysis that radiographic response to chemotherapy was correlated with malignant small bowel obstruction resolution $(\mathrm{OR}=6.81 ; 95 \%$ $\mathrm{CI}=1.68-27.85, P=0.007) .{ }^{19}$

This study did not find a separable long-term survival of surgical intervention in comparison with non-surgical treatment. However, $\mathrm{Smith}^{20}$ discovered that surgery improves survival in intestinal obstruction patients compared to non-surgical patients. The disparity may be explained, in part, by the selection criteria of the patients. They recruited patients mainly from the emergency department who urgently needed surgical intervention, while surgery categorized "elective" was excluded. In their

Table 3 Variables in the Final Equation. Variables Were Analyzed Through the Use of Logistic Regression Analysis with Forward LR Method. Prior Chemotherapy Was Transformed to Binary Data $(0=$ no, I=yes)

\begin{tabular}{|l|l|l|l|l|l|l|l|l|}
\hline & B & SE & Wals & df & Sig. & \multicolumn{2}{|l|}{ Exp (B) } & \multicolumn{2}{l|}{ Exp(B) 95\% CI } & Lower & Upper \\
\hline Prior chemotherapy & & & & & & & & \\
PG-SGA scoring at discharge & 2.521 & 1.174 & 4.615 & $I$ & 0.032 & 12.443 & 1.247 & 124.132 \\
Constant & 0.392 & 0.211 & 3.459 & $I$ & 0.063 & 1.480 & 0.979 & 2.237 \\
\hline
\end{tabular}


study, it is very likely that the emergency patients were mostly non-tumor patients, which is in strong contrast with cancerous patients. As cancerous tissues grow in a competitive and destructive way, ${ }^{21}$ it is not surprising that their operative intervention could achieve a satisfactory outcome in such a non-malignant cohort. Based on this point and our finding of prior chemotherapy as a significant variable, we herein propose an apriori assumption that anti-tumor treatment is a crucial part in the management of malignant bowel obstruction. Although intuitive, the inclusion of anti-tumor treatment seems, to some extent, to be more important than the surgical intervention alone because tumor growth and invasiveness can further worsen the general status, a problem that cannot be solved through the application of local resection surgery alone in end-stage patients. However, the idea is by no means to negate the effect of other treatment options, rather it offers a new consideration complementing the mainstream choices. Evidence supporting the proposition includes that combined chemotherapy and surgery could significantly improve the overall survival in MBO patients compared with surgery alone. ${ }^{22}$ To this end, taking antitumor measures into consideration seems to represent an important means of elongating overall survival in the treatment of MBO patients.

There are several limitations from this study. In the first place, the inherent nature of the study design as a retrospective analysis makes it less credible to extend the current conclusion into other circumstances. To address this point, further investigations, especially large randomized prospective studies, are warranted. However, we believe that the pilot description and initial conclusions can indicate the direction for subsequent large samplesized studies of other scholars. In the second place, there is no sham control group in the cohort. Without sham control, it seems quite difficult to exclude the influence of factors other than the surgery conduct itself, including implication of psychological factors, impact of perioperative anesthetics, and additional application of postoperative drugs following surgery than non-surgery. ${ }^{23}$ Nonetheless, to perform sham surgery on the human body is often not in accordance with humanitarian regulations and ethics considerations. Thus, animal studies particularly with a sham surgery group are needed to obliterate the effect of other confounding factors related to surgery. Last but not least, the detailed molecular mechanisms comprising biological pathways and cellular components are not investigated from the current study and therefore remain obscure. To this end, basic medical experiments aiming to reveal molecular functions between disease and treatment should be conducted. The revealed information will be helpful for the discovery of future prognostic markers or even pharmaceutical intervention targets.

\section{Conclusion}

In summary, this study described the clinical features of patients with $\mathrm{MBO}$, and analyzed the survival-related factors of the surgery and conservative groups. The uncovered results might provide beneficial information for future large scale mechanistic in-depth investigations.

\section{Abbreviations}

MBO, malignant bowel obstruction; PG-SGA, patientgenerated subjective global assessment; KPS, Karnofsky performance score; VAS, visual analog scale; mFI, modified frailty index; ASA, the American Society of Anesthesiology; CCI, Charlson comorbidity index; SE, standard error; OR, odds ratio.

\section{Acknowledgments}

This work was supported by the Special project for clinical medicine development of Beijing Municipal Hospital Administration (No. ZYLX201839); National Key Research and Development Projects (2017YFC1309200). Kaiying Yu and Lihui Liu are co-first authors for this study.

\section{Disclosure}

The authors declare that there is no conflict of interest.

\section{References}

1. Lilley EJ, Cauley CE, Cooper Z. Using a palliative care framework for seriously Ill surgical patients: the example of malignant bowel obstruction. JAMA Surg. 2016;151:695-696. doi:10.1001/jamasurg. 2016.0057

2. Krouse RS. Malignant bowel obstruction. J Surg Oncol. 2019; 120:74-77. doi: $10.1002 /$ jso. 25451

3. Jatoi A, Podratz KC, Gill P, Hartmann LC. Pathophysiology and palliation of inoperable bowel obstruction in patients with ovarian cancer. J Support Oncol. 2004;2:323-334; discussion 334-327.

4. Tuca A, Guell E, Martinez-Losada E, Codorniu N. Malignant bowel obstruction in advanced cancer patients: epidemiology, management, and factors influencing spontaneous resolution. Cancer Manag Res. 2012;4:159-169. doi:10.2147/CMAR.S29297

5. Tran E, Spiceland C, Sandhu NP, Jatoi A. Malignant bowel obstruction in patients with recurrent ovarian cancer. Am J Hosp Palliat Care. 2016;33:272-275. doi:10.1177/1049909114566225

6. Bento JH, Bianchi ET, Tustumi F, et al. Surgical management of malignant intestinal obstruction: outcome and prognostic factors. Chirurgia. 2019;114:343-351. doi:10.21614/chirurgia.114.3.343 
7. Shinjo T, Kagami R. Radiological imaging change in a malignant bowel obstruction patient treated with octreotide. Support Care Cancer. 2009;17:753-755. doi:10.1007/s00520009-0608-4

8. Suri S, Gupta S, Sudhakar PJ, et al. Comparative evaluation of plain films, ultrasound and CT in the diagnosis of intestinal obstruction. Acta Radiol. 1999;40:422-428. doi:10.3109/02841859909177758

9. Ripamonti CI, Easson AM, Gerdes H. Management of malignant bowel obstruction. Eur J Cancer. 2008;44:1105-1115. doi:10.1016/ j.ejca.2008.02.028

10. Paul Olson TJ, Pinkerton C, Brasel KJ, Schwarze ML. Palliative surgery for malignant bowel obstruction from carcinomatosis: a systematic review. JAMA Surg. 2014;149:383-392. doi:10.1001/ jamasurg.2013.4059

11. Chi DS, Phaëton R, Miner TJ, et al. A prospective outcomes analysis of palliative procedures performed for malignant intestinal obstruction due to recurrent ovarian cancer. Oncologist. 2009;14:835-839. doi:10.1634/theoncologist.2009-0057

12. Ripamonti C, Twycross R, Baines M, et al. Clinical-practice recommendations for the management of bowel obstruction in patients with end-stage cancer. Support Care Cancer. 2001;9:223-233. doi:10.100 7/s005200000198

13. Anthony T, Baron T, Mercadante S, et al. Report of the clinical protocol committee: development of randomized trials for malignant bowel obstruction. J Pain Symptom Manage. 2007;34:S4959. doi:10. 1016/j.jpainsymman.2007.04.011

14. Bauer J, Capra S, Ferguson M. Use of the scored patient-generated subjective global assessment (PG-SGA) as a nutrition assessment tool in patients with cancer. Eur J Clin Nutr. 2002;56:779-785. doi:10.1038/sj. ejcn.1601412
15. Charlson ME, Pompei P, Ales KL, MacKenzie CR. A new method of classifying prognostic comorbidity in longitudinal studies: development and validation. J Chronic Dis. 1987;40:373-383. doi:10.1016/ 0021-9681(87)90171-8

16. Lakomkin N, Zuckerman SL, Stannard B, et al. Preoperative risk stratification in spine tumor surgery: a comparison of the modified charlson index, frailty index, and ASA score. Spine. 2019;44: E782e787. doi:10.1097/BRS.0000000000002970

17. Langley GB, Sheppeard H. The visual analogue scale: its use in pain measurement. Rheumatol Int. 1985;5:145-148. doi:10.1007/BF00541514

18. Milstein JM, Cohen ME, Sinks LF. The influence and reliability of neurologic assessment and Karnofsky performance score on prognosis. Cancer. 1985;56:1834-1836.

19. Chouhan J, Gupta R, Ensor J, et al. Retrospective analysis of systemic chemotherapy and total parenteral nutrition for the treatment of malignant small bowel obstruction. Cancer Med. 2016;5:239-247. doi:10.1002/cam4.587

20. Smith BP. Surgery improves survival among patients with intestinal obstruction. Temple University. 2010. Master of Science.

21. Dai X, Xiang L, Li T, Bai Z. Cancer hallmarks, biomarkers and breast cancer molecular subtypes. J Cancer. 2016;7:1281-1294. doi:10.7150/ jca. 13141

22. Higashi H, Shida H, Ban K, et al. Factors affecting successful palliative surgery for malignant bowel obstruction due to peritoneal dissemination from colorectal cancer. Jpn $J$ Clin Oncol. 2003;33:357-359. doi:10.1093/jjco/hyg061

23. Rao B, Shi H. Malignant bowel obstruction: a struggle of technology, emotion and hope. Electron J Metab Nutr Cancer. 2017;4:136-143.

\section{Publish your work in this journal}

Cancer Management and Research is an international, peer-reviewed open access journal focusing on cancer research and the optimal use of preventative and integrated treatment interventions to achieve improved outcomes, enhanced survival and quality of life for the cancer patient.
The manuscript management system is completely online and includes a very quick and fair peer-review system, which is all easy to use. Visit http://www.dovepress.com/testimonials.php to read real quotes from published authors. 